\title{
Correlation between gut microbiota diversity and psychogenic erectile dysfunction
}

\author{
Qiang Geng ${ }^{1,2 \#}$, Shaofeng Chen ${ }^{1,2 \#}$, Yuan Sun ${ }^{1,2}$, Yu Zhao ${ }^{1,2}$, Zhong Li $^{1,2}$, Fu Wang ${ }^{3}$, Guojin Yu ${ }^{3}$, \\ Xiuchuan Yan ${ }^{4}$, Jiwei Zhang \\ ${ }^{1}$ Department of Andrology, First Teaching Hospital of Tianjin University of Traditional Chinese Medicine, Tianjin, China; ${ }^{2}$ Department of \\ Andrology, National Clinical Research Center for Chinese Medicine Acupuncture and Moxibustion, Tianjin, China; ${ }^{3}$ Department of Andrology, \\ Xiyuan Hospital, Chinese Academy of Traditional Chinese Medicine, Beijing, China; ${ }^{4}$ Nexbrio Medical Laboratory, Chengdu, China \\ Contributions: (I) Conception and design: All authors; (II) Administrative support: None; (III) Provision of study materials or patients: None; (IV) \\ Collection and assembly of data: None; (V) Data analysis and interpretation: All authors; (VI) Manuscript writing: All authors; (VII) Final approval of \\ manuscript: All authors. \\ "These authors contributed equally to this work as co-first authors. \\ Correspondence to: Jiwei Zhang. Department of Andrology, Xiyuan Hospital, Chinese Academy of Traditional Chinese Medicine, Beijing, China. \\ Email: zhangjiweixyyy@163.com.
}

Background: To analyze the distribution of gut microbiota in erectile dysfunction (ED) patients and explore the relationship between the diversity of gut microbiota and psychogenic ED.

Methods: Stool specimen were collected from 30 patients with ED and 30 healthy persons (healthy donors, HDs) and analyzed Paired end (PE) 300 sequencing on V3-V4 region sequences of bacterial 16S rRNA gene by using Illumina's Miseq platform, whereby sequencing results were analyzed to assess differences in species composition and diversity. The analysis comprised five modules: sequencing data quality control, operational taxonomic units (OTU) species clustering and annotation, alpha diversity, beta diversity and the use of $t$-tests and analysis of linear discriminant analysis effect size (LEfSe) differences.

Results: The International Index of Erectile Function (IIEF-5) score ranged between 8 and 21. The scores of $E D$ patients were $\geq 11$ and $\leq 20$, and the mean value was $15.67 \pm 2.94$. The flora diversity in the group of ED patients was significantly different from that of HDs $(\mathrm{P}<0.01)$, with the ED group having low bacterial diversity. There were no significant differences in the genus level between the ED and HD group, and abundant bacteria (TOP10) and core flora (90\%). Comparison of total flora (the abundance $>1 \%$ ) display, Alloprevotella genera showed differences, whereby Alloprevotella was only be identified in the HD group. Erectile dysfunction and HD showed good separation and clustering respectively in principal component analysis, showing significant differences in two kinds of microflora. T-tests showed that six species were significantly different, and that in the ED group, streptococci and Subdoligranulum were significantly increasing, and Prevotella sp.9, Blautia, Lachnospiraceae NK4A136 groups and Roseburia were significantly lower. Analysis using LEfSe analysis revealed 24 species were significantly different between ED and HD groups.

Conclusions: When gene sequencing was performed of ED and HD specimens, the microbial community structure and diversity showed significant differences, suggesting that ED specimen had lower gut microbiota diversity.

Keywords: Erectile dysfunction (ED); gut microbiota

Submitted Sep 16, 2021. Accepted for publication Nov 10, 2021.

doi: $10.21037 /$ tau-21-915

View this article at: https://dx.doi.org/10.21037/tau-21-915 


\section{Introduction}

A large number of microorganisms live in the human intestinal tract. The normal gut microbiota is the natural barrier of the human body and plays an important part in maintaining human health (1). The normal gut microbiota in the human body is mainly composed of firmicutes, Bacteroidetes, proteobacteria, and actinomycetes. According to the impact on human health, gut microbiota can be divided into three categories: symbiotic bacteria, opportunistic bacteria, and pathogenic bacteria, which are dynamically balanced to maintain the homeostasis of the intestinal environment (2). Intestinal beneficial bacteria (i.e., probiotics) include bifidobacteria, lactobacillus, and so on. Harmful bacteria include Escherichia coli and enterococcus (3). Changes in the internal and external environment of the body can affect the structure of gut microbiota, resulting in gut microbiota imbalance (4), which can lead to inflammatory bowel disease (5), irritable bowel syndrome (6), non-alcoholic liver disease (7), viral hepatitis (8), metabolic syndrome (9), and other diseases, aggravation, and rapid progression. Sexual function is a complex phenomenon affected by sex hormones, psychological and neurological factors, hemodynamics, hormonal disorders, obesity, stress, anxiety, hypertension, diabetes and so on. It had been reported that gut microbiota was related to endocrine, psychological factors and metabolic syndrome. The causes of ED was generally believed as vascular, neurologic, psychological, and hormonal factors. Conditions commonly associated with ED include diabetes mellitus, hypertension, hyperlipidemia, obesity, testosterone deficiency. Performance anxiety and relationship issues are common psychological causes $(10,11)$. Therefore, it might be hypothesized that there is a correlation between ED and gut microbiota distribution, and the distribution of gut microbiota was different between the HD and ED populations. To test this hypothesis, this study compared the gut microbiota diversity of patients with ED to that of a normal control group. We present the following article in accordance with the MDAR reporting checklist (available at https://dx.doi.org/10.21037/tau-21-915).

\section{Methods}

\section{Research objects}

ED group: 30 ED patients with an average age of $29.33 \pm 2.73$ years were seen at the First Teaching Hospital of Tianjin University of Traditional Chinese Medicine and
Xiyuan Hospital, Chinese Academy of Traditional Chinese Medicine from October 2018 to October 2019. Healthy donor group: 30 healthy volunteers aged $20-40$ years with an average age of $29.17 \pm 2.66$ years. There was no statistically significant difference in age between the two groups $(\mathrm{P}>0.05)$.

\section{Diagnostic criteria for $E D$}

In the ED group, the diagnostic criteria of mild to moderate ED had to be satisfied. The International Index of Erectile Function (IIEF-5) score ranged between 8 and 21, and the diagnosis was consistent between two or more attending physicians. In the HD group, male physicians with a title of associate chief physician or above were administered the IIEF-5 and scored between 22 and 25 .

\section{Inclusion criteria}

Married men aged 20-40 years with regular sexual partners, who agreed to participate in the study; normal genital and secondary sexual characteristics; no acute or chronic gastrointestinal diseases; no history of severe hypertension, myocardial infarction or other serious cardiovascular disease, diabetes, blood, kidney or liver disease; no history of infectious diseases such as tuberculosis, viral hepatitis, and acquired immune deficiency syndrome (AIDS); did not participate in other clinical trials in the last 3 months; did not use antibiotics or steroid drugs; and did not eat probiotics or other microbiological preparations. In the ED group, the diagnostic criteria of mild to moderate ED had to be satisfied. The International Index of Erectile Function (IIEF-5) score ranged between 8 and 21, and the diagnosis was consistent between two or more attending physicians. In the HD group, male physicians with a title of associate chief physician or above were administered the IIEF-5 and scored between 22 and 25. The study was approved by the Ethics Committee of the Xiyuan Hospital of Chinese Academy of Traditional Chinese Medicine (No. 2018XLA064-2), and study implementation strictly followed the approved protocol. Informed consent was taken from all individual participants. All procedures performed in this study involving human participants were in accordance with the Declaration of Helsinki (as revised in 2013).

\section{Exclusion criteria}

Obvious primary diseases and/or complications of the heart, liver, kidney and nervous system; other sexual dysfunction, 
such as decreased libido, orgasm suppression or no orgasm, ejaculation suppression, or no ejaculation; female partners have significant sexual dysfunction, such as decreased libido or painful sexual intercourse; drug abuse within the last 2 years; acute and chronic inflammation of the reproductive system; penis deformities, genital deformities that directly impair erectile function; and presence of serious mental disorders.

\section{Collection of samples}

The two groups were instructed to sample about $50 \mathrm{~g}$ of fresh stool specimens before eating food in the morning, place it into a special specimen bottle, and quickly put the bottle into an ice box for preservation at $-80{ }^{\circ} \mathrm{C}$ (Siemens, Germany). All samples were kept in dry ice and sent to Nexbrio Medical Laboratory for gene sequencing and data analysis.

\section{Gene sequencing and data analysis}

(I) DNA extraction: depending on the sample type, the corresponding kit was used to extract the sample DNA and remove other non-DNA substances from the sample. (II) DNA quality control: $0.8-1 \%$ agarose gel was used to detect the integrity, degradation, and degradation degree of DNA; Q-bit instruments and matching DNAspecific dyes were used to detect the DNA concentration. The A260/A280 and A260/A230 of DNA were detected using nanodrop (Thermo, USA) to determine the purity of DNA. (III) Library construction: V3 and V4 regions were amplified using the universal primers of $16 \mathrm{~S}$ rRNA gene. The polymerase chain reaction (PCR) (Thermo, America) amplification products were purified, and the redundant primers and various components in the PCR reaction system were removed. The purified product was supplemented using PCR, the terminals of the purified product were added to the connector for sequencing, and the index label sequence attached to denote each sample. The second round of PCR amplification products was purified, and the redundant primers and various components in the PCR reaction system were removed. (IV) Library quality inspection and pretreatment before sequencing: Q-BIT was used to identify the concentration of each library, and all libraries were mixed according to equal molality; fluorescence quantitative PCR was used to detect the molality of the mixed library. The total library of sodium hydroxide treatment was single-stranded DNA. (V) Clustering: using Illumina's Miseq sequencer, singlestranded DNA generated was hybridized with singlestranded sequence of flow cell, and then clusters of singlestranded DNA were formed on the flow cell by bridging PCR amplification. Finally, the DNA in the cluster was converted into single-stranded DNA and then pairedend (PE) sequencing was undertaken. (VI) Sequencing: using Miseq, the base sequence of each cluster on the flow cell was observed using simultaneous synthesis and sequencing. (VII) Disinformation analysis: Raw Data were firstly quality controlled, filtered, spliced, and removed to obtain clean data, then the effective data were analyzed for operational taxonomic units (OTU) and species classifications. According to the OTUs clustering results, species annotation was made on the representative sequence of each OTU to obtain the analogous species information and species-based abundance distribution. At the same time, OTUs were tested for relative abundance, and an alpha diversity calculation was undertaken, to obtain species richness and uniformity information in samples, as well as differences between different samples or groups. To further explore the differences in community structure between the group samples, $t$-tests, linear discriminant analysis effect size (LEfSe), and other statistical analysis methods were utilized to test the significance of the differences in species composition and community structure between the ED and the HD groups. A P value $<0.05$ was considered as indicating statistical significance.

\section{Statistical analysis}

Data were expressed as mean \pm SEM. A one way analysis of variance (ANOVA) was used to detect statistical significance. Once the significant difference was detected, a $t$-test was conducted using SPSS 19.0 software (IBM Co., Armonk, USA). $\mathrm{P}$ values less than 0.05 were statistical difference, and $\mathrm{P}$ values less than 0.01 were considered significant.

\section{Results}

\section{IIEF-5 score in ED group}

In the ED group, the diagnostic criteria of mild to moderate ED were satisfied. The IIEF-5 score was between 8 and 21 . The scores of ED patients were $\geq 11$ and $\leq 20$, and the mean value was $15.67 \pm 2.94$. 


\section{Data preprocessing and quality control}

The total DNA of the sequenced samples was tested. The concentration and purity of 49 samples (ED: 26, HD: 23) met the sequencing requirements of the Illumina MiSeq platform.

\section{Analysis of bacterial diversity in fecal samples}

\section{Analysis of bacterial flora composition of samples}

According to OTU annotation results, histograms of relative abundance of species were generated for each sample at different classification levels, which visually displayed the bacterial flora of each sample at different classification levels. Figures 1,2 show stacked histograms of bacteria with relative abundance greater than $1 \%$ in each sample at the classification level of genera. The abscissa is the sample name, and the ordinate is the relative abundance ratio of corresponding bacteria. " $\mathrm{F}_{-}{ }^{*}$ " means that it cannot be annotated to genus in a biological classification but can be annotated to family or other classification level.

There was no difference between the genera of the ED-HD group, indicating that there was no statistically significant difference between the high abundance bacteria (top 10) and the core bacteria (90\%) of the ED-HD group. However, of the total intergroup flora $(>1 \%)$ between groups, Alloprevotella was statistically different, in that Alloprevotella was identified only in the HD group.

\section{Alpha diversity analysis}

Alpha diversity refers to the microbial community diversity in a specific region or ecological environment, which is a comprehensive index reflecting richness and uniformity. The alpha diversity analysis of a single sample can reflect the richness and diversity of the microbial community in the sample. Figure 3 shows that the ED group had a relatively low Shannon diversity coefficient (HD group $=5.741$, ED group $=4.982$ ), indicating that the ED group had lower bacterial diversity $(\mathrm{P}=0.00074 \mathrm{X}<0.01)$.

\section{Principal component analysis (PCoA) based on Bate diversity \\ Principal component analysis (also referred to as principal co-ordinates analysis) is used to study the similarity or heterogeneity of sample community composition. The closer the distance of the sample, the higher the similarity of the community structure, otherwise the structure indicates the larger difference of the community. Figure 4 is the}

PCoA conducted by calculating the unweighted UniFrac distance using the relationship between OTU systems. The $\mathrm{x}$-coordinate represents one principal component, the $\mathrm{y}$-coordinate represents another principal component, and the percentage represents the contribution value of the principal component to the difference of samples. Each point in the figure represents a sample, and samples of the same group are represented by the same color. The results show that the ED group (blue) and the HD group (red) are clustered, and the two groups are clearly separated, indicating that the structure of the ED-HD group is different.

\section{Comparison of relative abundance between groups of all strains}

Statistical analysis specifically identified the genera with significant differences in the abundance change between groups, and the enrichment of the genera with significant differences between groups was obtained. The size of the differences between groups was compared to determine whether the community structure difference between groups was statistically significant.

\section{t-test}

The $t$-test was used to detect significant differences between groups at the level of genera $(\mathrm{P}<0.05)$. Results (Table 1 and Figures 5,6) show six species were significantly different between groups, streptococcus and Subdoligranulum were increased in the ED group compared with the HD group, while in the Prevotella, Blautia, Lachnospiraceae NK4A136 group, and Roseburia were decreased compared with the HD group.

\section{LEfSe}

To find the specific bacterial genera of ED, LEfSe analysis was used to compare the different bacterial genera between the two groups. The results (Figure 7) showed that the absolute value of the linear discriminant analysis (LDA) scores of 24 bacterial genera was above 2. It is known that the Lachnospiraceae NK4A136 group and Prevotella_9 have the greatest influence on the difference between groups.

Comments:

(I) LDA is a supervised dimension reduction method, while PCA is an unsupervised dimension reduction method.

(II) LDA dimension reduction can be reduced to the dimension of category number K-1 at most while 


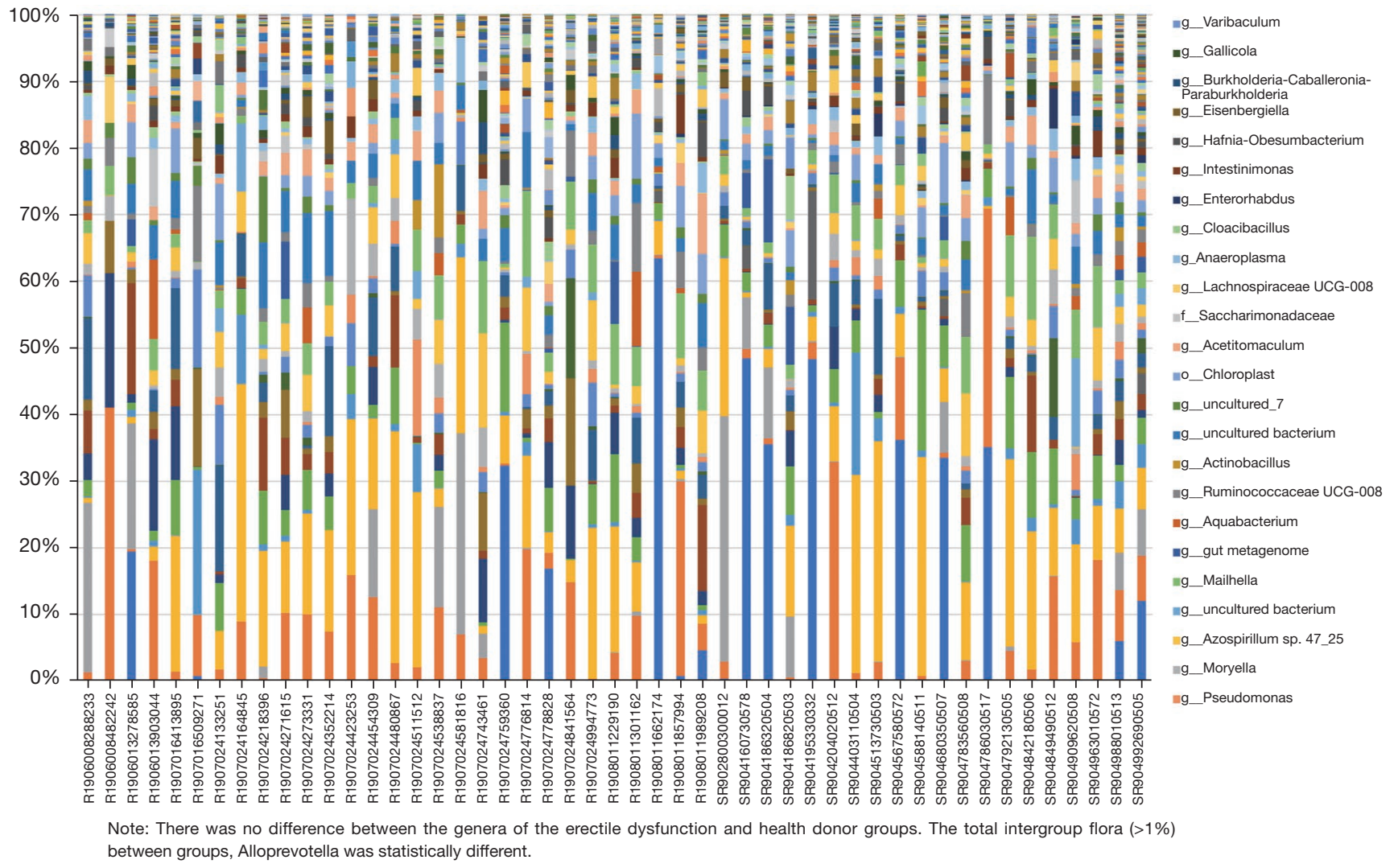

Figure 1 Distribution of abundance bacteria and core bacteria in erectile dysfunction and health donors groups.

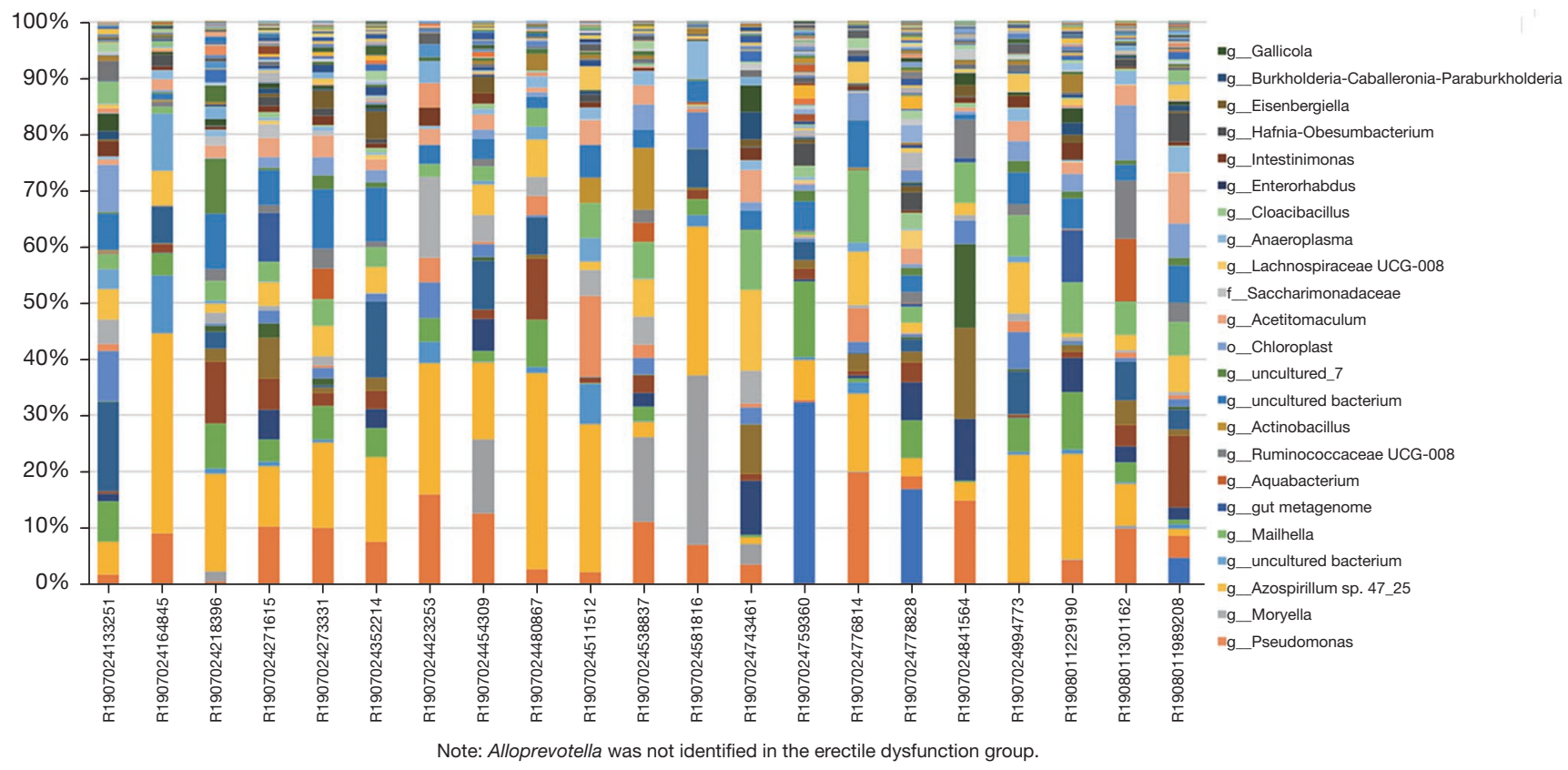

Figure 2 The distribution of abundance bacteria and core bacteria in the erectile dysfunction groups. 


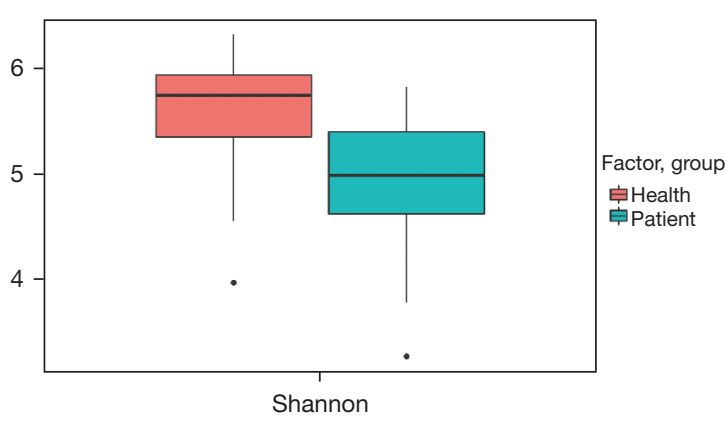

Note: Compared with the health donor group, the erectile dysfunction group had lower bacterial diversity.

Figure 3 The box diagram of Shannon Index box diagram (Alpha Diversity).

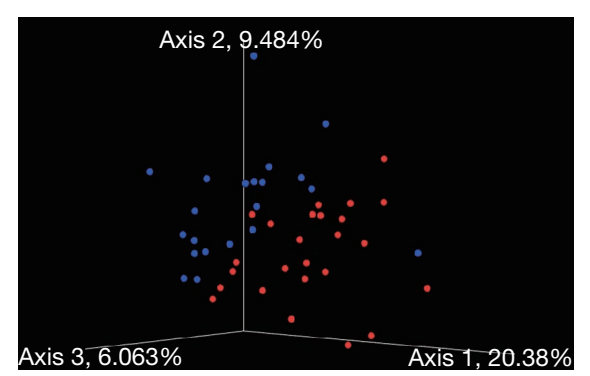

Note: Bacterial structure of erectile dysfunction-health donor group was different.

Figure 4 PCoA analysis between the erectile dysfunction and health donor groups.

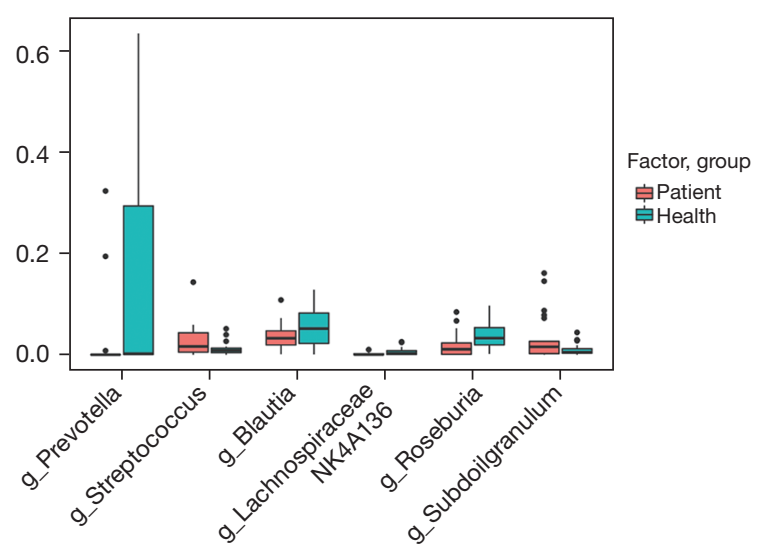

Figure 5 Boxplot figures of significant differences in species between erectile dysfunction and health donor groups.

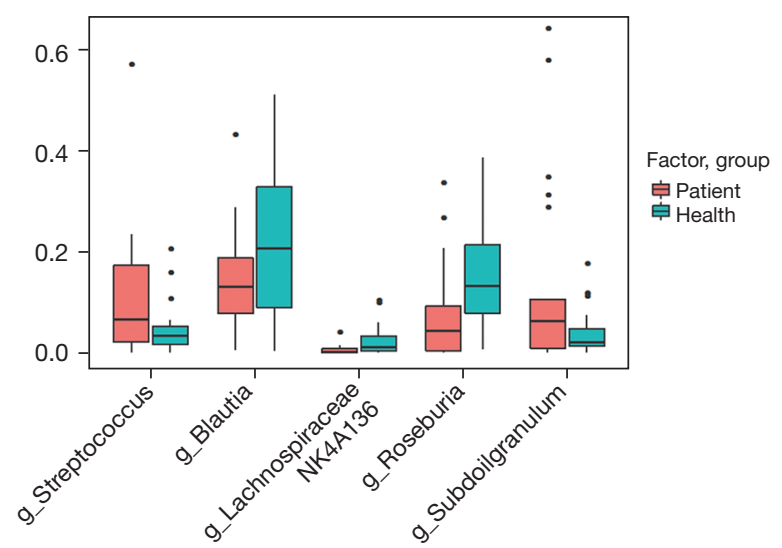

Figure 6 Boxplot figures of significant differences species between erectile dysfunction and health donor groups (except Prevotella).

Table 1 Species that were significantly different in the erectile dysfunction and health donor groups

\begin{tabular}{lcc}
\hline Class & P value & Significance \\
\hline g_Prevotella 9 & 0.02558353 & $*$ \\
g_Streptococcus & 0.043744221 & $*$ \\
g_Blautia & 0.048518626 & $*$ \\
g_LachnospiraceaeNK4A136 group & 0.006742402 & $*$ \\
g_Roseburia & 0.017978557 & $*$ \\
g_Subdoligranulum & 0.036087622 & $*$ \\
\hline
\end{tabular}

${ }^{*}, \mathrm{P}<0.05 ;{ }^{*}, \mathrm{P}<0.01$. 


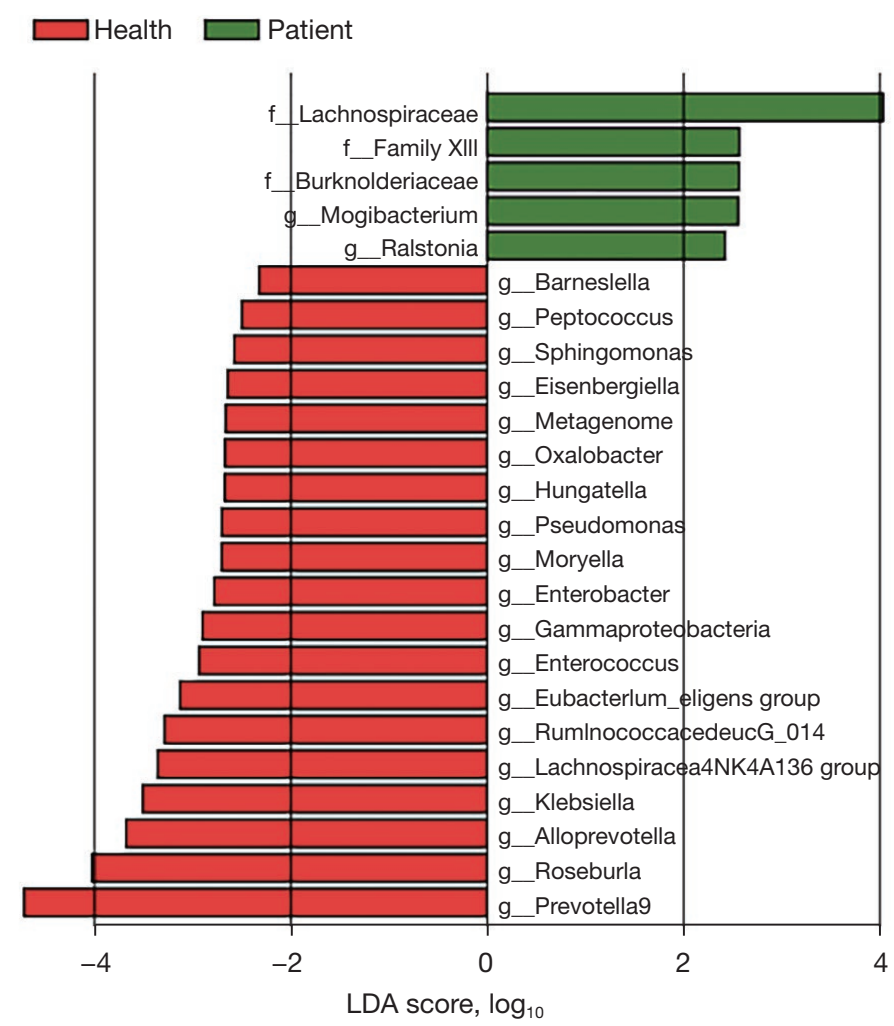

Figure 7 Linear discriminant analysis value distribution histogram.

PCA cannot.

(III) In addition to dimension reduction, LDA can also be used for classification.

(IV) LDA selects the projection direction with the best classification performance, while PCA selects the direction with the greatest variance for the projection of sample points.

The main advantages of LDA algorithm are as follows:

(I) Prior knowledge of categories can be used in dimension reduction, while unsupervised learning such as PCA cannot use prior knowledge of categories.

(II) LDA is better than PCA when the sample classification information depends on mean value instead of variance.

The main disadvantages of LDA algorithm are as follows:

(I) LDA is not suitable for dimensionality reduction of non-Gaussian distribution samples, and PCA also has this problem.

(II) LDA dimension reduction can be reduced to the dimension of category number K-1 at most. If the dimension reduction is greater than $\mathrm{K}-1, \mathrm{LDA}$ cannot be used. There are some evolutionary versions of the LDA that can circumvent this problem.

(III) When the sample classification information depends on variance instead of mean value, LDA has a poor effect on dimension reduction.

(IV) LDA may overfit the data.

\section{Discussion}

Normal erectile function is a complex phenomenon affected by sex hormones, psychological and neurological factors, hemodynamics, hormonal disorders, obesity, stress, anxiety, hypertension, and diabetes. The hormone was the important influenced factor for ED. The gut microbiotas can regulate the endocrine system and affect androgen levels $(12,13)$. Markle et al. confirmed that the intestinal microbiota of sterile mice was regulated by microbiota, and the intestinal microbiota of normal male mice was implanted into female nude mice, which resulted in the sustained increase of testosterone levels in females and the regulation of their autoimmunity. It has also been shown 
that when female mice were treated with normal intestinal microbiota obtained from donor male mice in the context of impaired reproductive capacity, testosterone levels were significantly increased (13). Microbiota-based therapies have also been used for diseases of the immune system, such as type 1 diabetes, which is linked to abnormal levels of sex hormones (14). In a well-designed study, Yurkovetskiy et al. showed that germ-free 5-week-old mice had higher plasma testosterone concentrations than nonpathogen free (SPF) mice, whereas SPF mice had elevated testosterone levels after 12 weeks. In addition, mice transplanted with filamentous bacteria or proteobacteria similar to Shigella escherichia coli showed higher serum testosterone concentrations at 12 weeks of age than mice without bacteria. However, the probiotic mixture did do not increase testosterone levels (15). Consistent with the above, the microbiome can positively and negatively regulate sex hormone levels based on host physiology, bacterial strains, and other factors. These findings strongly indicate that the gut microbiota can improve sexual function by regulating sex hormone levels. Reiter probiotics had positive effects on testicular size and serum testosterone levels in aging mice (16). Thus, it can be observed that using probiotics to regulate human microbiota is another method to improve sexual ability. Normal erectile function depends on normal functional penile blood vessels and cavernous blood vessels. It has been found that the growth of gut microbiota in ED patients with diabetes was inhibited, the number of probiotics was significantly reduced, but the number of opportunistic pathogens was increased (17). Cho et al. found that serum timethylamine $\mathrm{N}$-oxide (TMAO) was associated with intestinal germicides (18). Seldin $e t$ al. found that TMAO could promote vascular endothelial inflammation and enhance smooth muscle cell proliferation, eventually leading to vascular wall fibrosis and vascular function destruction (19). Sun et al. found that TMAO could lead to vascular endothelial dysfunction through reactive oxygen species (ROS) mediated oxidative stress, as well as increased adhesion of monocytes in human umbilical cord vessels and endothelial repair dysfunction (20). Therefore, this study reviewed the association between gut microbiota distribution and ED.

Numerous studies have demonstrated that the pathogenesis of ED is related to the inflammatory response. Streptococci mostly cause inflammation, and they include Group A Streptococcus (GAS), which is A gram-positive pathogen and can cause a range of diseases, including deadly invasive infections. A study by Hashizume-Takizawa et al. showed that Streptococcus hemoglobin and Streptococcus are associated with systemic diseases such as infective endocarditis and abscess and increased expression levels of the inflammation-related factor IL-1 mRNA, when mice were attacked by Streptococcus bemoglobin (21). Roxella is comprised of gram-positive anaerobes and is one of the common bacteria that produce short-chain fatty acids (SCFAs), especially butyric acid, which affects colon motility and has immunomaintenance and anti-inflammatory effects (22). The results of this study showed that streptococci were upregulated and rosteria were downregulated, both streptococci and rosteria were associated with the inflammatory response, and ED was involved in inflammation and, therefore, streptococcus and rosteria were associated with ED.

Obesity increases the likelihood of ED (23). Studies have further shown a link between human gut flora and obesity. Modification of the characterization of Roxella may affect various metabolic pathways, resulting in various diseases such as irritable bowel syndrome, obesity, type 2 diabetes, neurological diseases, and allergies (22). Miao et al. confirmed that the rats with metabolic syndrome had obvious gut microbiota imbalance, and the content of opportunistic pathogens such as streptococcus increased (24). The results of this study showed that the number of streptococci in ED patients was upregulated, while the number of rosteria was downregulated.

In this study, patients were selected to detect the fecal bacterial diversity of the functional ED and HD groups. Results of alpha diversity analysis showed that the ED group had lower bacterial diversity. It was further confirmed that there were significant differences between the two groups at the colony and genus level. Level analysis also showed that the number of streptococci and Subdoligranulum (rare small cocci) genera in the ED group increased, while the number of Prevotella_9, Blautia, Lachnospiraceae NK4A136 group, and Roseburia decreased.

In summary, ED is associated with intestinal microbial diversity in men. Gut microbiota can regulate male erectile function by regulating hormone levels, inflammatory mediators, and other factors. Gut microbiota is a promising and promising option for the preventing and treatment of $\mathrm{ED}$ in men in the future. For preventing ED, healthy diet, regular work and rest, relaxed mental stress are necessary. And intestinal probiotics can be supplemented in moderation. 


\section{Acknowledgments}

Funding: The study was supported by the China Postdoctoral Foundation (No. 2016M601236).

\section{Footnote}

Reporting Checklist: The authors have completed the MDAR reporting checklist. Available at https://dx.doi. org/10.21037/tau-21-915

Data Sharing Statement: Available at https://dx.doi. org/10.21037/tau-21-915

Conflicts of Interest: All authors have completed the ICMJE uniform disclosure form (available at http://dx.doi. org/10.21037/tau-21-915). The authors have no conflicts of interest to declare.

Ethical Statement: The authors are accountable for all aspects of the work in ensuring that questions related to the accuracy or integrity of any part of the work are appropriately investigated and resolved. All procedures performed in this study involving human participants were in accordance with the Declaration of Helsinki (as revised in 2013). The study was approved by the Ethics Committee of the Xiyuan Hospital of Chinese Academy of Traditional Chinese Medicine (No. 2018XLA064-2), and study implementation strictly followed the approved protocol. Informed consent was taken from all individual participants.

Open Access Statement: This is an Open Access article distributed in accordance with the Creative Commons Attribution-NonCommercial-NoDerivs 4.0 International License (CC BY-NC-ND 4.0), which permits the noncommercial replication and distribution of the article with the strict proviso that no changes or edits are made and the original work is properly cited (including links to both the formal publication through the relevant DOI and the license). See: https://creativecommons.org/licenses/by-nc-nd/4.0/.

\section{References}

1. Sittipo P, Lobionda S, Lee YK, et al. Intestinal microbiota and the immune system in metabolic diseases. J Microbiol 2018;56:154-62.

2. Lin H, Liu T, Li X, et al. The role of gut microbiota metabolite trimethylamine $\mathrm{N}$-oxide in functional impairment of bone marrow mesenchymal stem cells in osteoporosis disease. Ann Transl Med 2020;8:1009.

3. Janssen AW, Kersten $S$. The role of the gut microbiota in metabolic health. FASEB J 2015;29:3111-23.

4. Li Y, Liu T, Yan C, et al. Diammonium Glycyrrhizinate Protects against Nonalcoholic Fatty Liver Disease in Mice through Modulation of Gut Microbiota and Restoration of Intestinal Barrier. Mol Pharm 2018;15:3860-70.

5. Xu P, Becker H, Elizalde M, et al. Intestinal organoid culture model is a valuable system to study epithelial barrier function in IBD. Gut 2018;67:1905-6.

6. Liu Y, Zhang L, Wang X, et al. Similar Fecal Microbiota Signatures in Patients With Diarrhea-Predominant Irritable Bowel Syndrome and Patients With Depression. Clin Gastroenterol Hepatol 2016;14:1602-11.e5.

7. Valencia-Rodríguez A, Vera-Barajas A, Barranco-Fragoso $\mathrm{B}$, et al. New insights into the association between nonalcoholic fatty liver disease and atherosclerosis. Ann Transl Med 2019;7:S300.

8. Szereday L, Meggyes M, Halasz M, et al. Immunological changes in different patient populations with chronic hepatitis $\mathrm{C}$ virus infection. World J Gastroenterol 2016;22:4848-59.

9. Lin L, Wen ZB, Lin DJ, et al. Correlations between microbial communities in stool and clinical indicators in patients with metabolic syndrome. World J Clin Cases 2018;6:54-63.

10. Hatzimouratidis K, Amar E, Eardley I, et al. European Association of Urology. Guidelines on male sexual dysfunction: erectile dysfunction and premature ejaculation. Eur Urol 2010;5:804-14.

11. Lombardi G, Nelli F, Celso M, et al. Treating erectile dysfunction and central neurological diseases with oral phosphodiesterase type 5 inhibitors. Review of the literature. J Sex Med 2012;9:970-85.

12. Clarke G, Stilling RM, Kennedy PJ, et al. Minireview: gut microbiota:the neglected endocrine organ. Mol Endocrinol 2014;28:1221-38.

13. Markle JG, Frank DN, Mortin-Toth S, et al. Sex differences in the gut microbiome drive hormone-dependent regulation of autoimmunity. Science 2013;339:1084-8.

14. Markle JG, Frank DN, Adeli K, et al. Microbiome manipulation modifies sex-specific risk for autoimmunity. Gut Microbes 2014;5:485-93.

15. Yurkovetskiy L, Burrows M, Khan AA, et al. Gender bias in autoimmunity is influenced by microbiota. Immunity 2013;39:400-12.

16. Everard A, Matamoros S, Geurts L, et al. Saccharomyces 
boulardii administration changes gut microbiota and reduces hepatic steatosis, low-grade inflammation, and fat mass in obese and type 2 diabetic $\mathrm{db} / \mathrm{db}$ mice. $\mathrm{mBio}$ 2014;5:e01011-14.

17. Li H, Qi T, Huang ZS, et al. Relationship between gut microbiota and type 2 diabetic erectile dysfunction in Sprague-Dawley rats. J Huazhong Univ Sci Technolog Med Sci 2017;37:523-30.

18. Cho CE, Taesuwan S, Malysheva OV, et al. Trimethylamine-N-oxide (TMAO) response to animal source foods varies among healthy young men and is influenced by their gut microbiota composition: A randomized controlled trial. Mol Nutr Food Res 2017;61. doi: 10.1002/mnfr.201600324.

19. Seldin MM, Meng Y, Qi H, et al. Trimethylamine N-Oxide Promotes Vascular Inflammation Through Signaling of Mitogen-Activated Protein Kinase and Nuclear Factor- $\kappa B$. J Am Heart Assoc 2016;5:002767.

20. Sun X, Jiao X, Ma Y, et al. Trimethylamine N-oxide induces inflammation and endothelial dysfunction in

Cite this article as: Geng Q, Chen S, Sun Y, Zhao Y, Li Z, Wang F, Yu G, Yan X, Zhang J. Correlation between gut microbiota diversity and psychogenic erectile dysfunction. Transl Androl Urol 2021;10(12):4412-4421. doi: 10.21037/tau-21-915 human umbilical vein endothelial cells via activating ROSTXNIP-NLRP3 inflammasome. Biochem Biophys Res Commun 2016;481:63-70.

21. Hashizume-Takizawa T, Yamaguchi Y, Kobayashi R, et al. Oral challenge with Streptococcus sanguinis induces aortic inflammation and accelerates atherosclerosis in spontaneously hyperlipidemic mice. Biochem Biophys Res Commun 2019;520:507-13.

22. Tamanai-Shacoori Z, Smida I, Bousarghin L, et al. Roseburia spp.: a marker of health? Future Microbiol 2017;12:157-70.

23. Rowland DL, McNabney SM, Mann AR. Sexual Function, Obesity, and Weight Loss in Men and Women. Sex Med Rev 2017;5:323-38.

24. Miao P, Li J, Zhou F, et al. Effect of resveratrol on main gut microbiota of rats with metabolic syndrome. Journal of Guangzhou University of Chinese Medicine 2020;37:711-5.

(English Language Editor: B. Meiser) 